\title{
Faut-il switcher de l'imatinib vers le dasatinib les leucémies myéloïdes chroniques qui ne sont pas en réponse optimale à trois mois ?
}

Virgnie Eclache

L es très bons résultats obtenus dans le traitement de la leucémie myéloïde chronique en phase chronique (LMC-PC) avec l'imatinib (IM), premier inhibiteur de tyrosine kinase (ITK), depuis le début des années 2000, sont incontestables. L'obtention d'une réponse moléculaire précoce (RMP) définie par un taux de transcrit BCR-ABL1 ( $\leq 10 \%)$ après trois à six mois de traitement est un bon indicateur de réponse moléculaire profonde (RM4.5 ou BCR-ABL1 $\leq 0,0032 \%$ ), d'une meilleure survie sans progression (SSP) et d'une meilleure survie globale (SG) [1]. Cependant, un tiers des patients ne sont pas en réponse optimale après trois mois de traitement par IM, ce qui constitue un signal de vigilance et pose la question d'un changement de traitement pour un ITK de deuxième génération. DASCERN est une étude prospective randomisée qui vise à évaluer la pertinence d'un changement précoce de traitement par du dasatinib (Dasa) pour les patients présentant une LMC-PC en réponse non optimale après trois mois d'IM. Cortes et al. [2] présentent les résultats de cette étude avec au moins deux ans de recul.

Les patients de plus de 18 ans en PC recevaient initialement $400 \mathrm{mg}$ d'IM durant trois mois, puis étaient testés quantitativement pour le transcrit BCR-ABL1. Ceux dont le transcrit était $\leq 10 \%$ étaient non éligibles car bons répondeurs. Les patients avec un taux de transcrit BCR-ABL1 $>10 \%$ étaient randomisés 2:1 pour recevoir du Dasa 100 mg/j ou continuer l'IM à la dose d'au moins $400 \mathrm{mg} / \mathrm{j}$. La randomisation réalisée dans les huit semaines après les trois mois de traitement initial était conçue pour répartir les différents stades de Sokal de façon équilibrée. Les patients étant en échec de traitement par l'IM pouvaient ensuite être switchés vers le Dasa sauf en cas de mutation de résistance au Dasa (T315I/A, F317L, V999L) documentée par un laboratoire centralisateur. La recherche de mutation de BCRABL1 était réalisée en cas d'échec de traitement, de réponse suboptimale ou de progression, à la fin du traitement ou avant chaque changement de traitement.

Les objectifs de l'étude étaient l'obtention d'une réponse moléculaire majeure (RMM) évaluée à 12 mois après le début du traitement (neuf mois après la randomisation) ainsi que le temps pour obtenir cette réponse, l'obtention d'une RM4.5, la SSP et la SG.

De septembre 2012 à novembre 2016, un total de 1126 patients ont été analysés, 260 avec un taux de BCR-ABL1 > $10 \%$, qui ont été randomisés : 174 dans le bras Dasa, 86 dans le bras IM. L'âge médian était de 37 ans (18-82) et $95 \%$ d'entre eux avaient moins de 65 ans. Les scores de Sokal uniformément répartis étaient bas (28\%), intermédiaires (30\%) élevés (24\%) ou inconnus $(18 \%)$. Les patients étaient majoritairement de sexe masculin (78\%), et d'origine asiatique $(73 \%), 84 \%$ avaient un bon performance status. Tous les patients avaient un transcrit M- BCR-ABL1 (b2a2 ou b3a2). Après 24 mois de suivi, 135 patients randomisés Dasa (79\%) et 68 patients du bras IM (79\%) étaient toujours traités. Après une moyenne de neuf mois (6-12) suivant la randomisation, 45/86 patients sous IM ont été switchés vers le Dasa dont 44 pour échec de traitement et un pour réponse suboptimale ; $71 \%$ étaient toujours traités après deux ans. Les interruptions, augmentations et réductions de traitement étaient respectivement de 44, 13 et $6 \%$ dans le bras Dasa et 43,12 et $8 \%$ dans le bras IM. Une RMM a été obtenue après 12 mois de traitement chez $29 \%$ des patients du groupe Dasa versus $13 \%$ du groupe IM ( $p=0,005)$. Au final, $66 \%$ des patients du bras Dasa et $67 \%$ de ceux du groupe IM ont obtenu une RMM, mais $30 \%$ des patients du bras IM ont reçu du Dasa en deuxième intention ; si on censure ces patients, 35/81 patients (41\%) ont obtenu une RMM sous IM seul. La RMM a été obtenue en moyenne après 14 mois de traitement par Dasa (95\%CI : 12-18), contre 20 mois avec l'IM seul (95\%CI : 14-26).

La réponse cytogénétique complète a été obtenue chez 147 (81\%) des patients du bras Dasa et 71 (83\%) du bras IM, et 29/45 patients du bras IM switchés ont obtenu secondairement une réponse cytogénétique complète sous Dasa.

La SSP à 24 mois était de $96 \%$ dans le bras Dasa contre 95 \% pour le bras IM ; en tenant compte du switch, la SSP à 24 mois était de $93 \%$ pour les patients ayant reçu secondairement du Dasa versus $98 \%$ pour ceux n'ayant reçu que l'IM. La SG montre les mêmes résultats que la SSP. La progression vers une phase accélérée ou blastique a été observée dans six cas du bras Dasa - dont deux après arrêt du Dasa pour cause de toxicité hématologique - et trois patients du bras IM dont deux après le changement de traitement. Des évènements indésirables ont été observés chez 141 (82\%) des patients dans le bras Dasa et 67 (78 \%) du bras IM et pour 38/45 patients ayant reçu du Dasa en deuxième intention (84\%). Un épanchement pleural a été observé chez 15 patients traités par Dasa (dont trois de grade $3 / 4$ ). 
Le traitement a été interrompu pour cause de toxicité chez 12 patients (7 \%) du bras Dasa, principalement pour toxicité hématologique de grade 3-4 (six patients) et épanchement pleural (cinq patients) et chez quatre (5\%) du bras IM, pour causes non hématologiques. La toxicité hématologique était comparable dans les deux bras : neutropénie de grade $3 / 4$ chez $12 \%$ des patients du bras Dasa et chez $16 \%$ du bras IM, et chez $29 \%$ des patients secondairement sous Dasa. Des complications cardiovasculaires ont été rapportées chez deux patients du bras Dasa et chez un patient du bras IM switchés vers le Dasa.

Les évènements indésirables graves non hématologiques les plus fréquents étaient des maux de tête dans le bras Dasa et une hypophosphatémie dans le groupe IM.

Bien que les résultats de cette étude montrent que l'obtention d'une RMM après 12 mois de traitement est supérieure dans le bras Dasa (29 versus $13 \%, \mathrm{p}=0,005$ ) et à 24 mois (64 versus $41 \%$ ), les taux de SSP et de SG ne sont pas significativement différents. Des études ont montré que l'obtention d'une réponse moléculaire majeure ou d'une réponse cytogénétique complètes était favorisée avec les ITK de deuxième génération mais il est difficile de montrer qu'un switch précoce pour des patients en réponse non optimale à trois mois donne une meilleure survie à long terme. Les résultats de cette étude demandent une réévaluation à long terme. Il est à noter que de nombreux effets secondaires sont survenus, dont certains ont eu pour conséquence des interruptions de traitement. Les cas d'épanchement pleuraux sous Dasa sont cependant rapportés en moins grand nombre (9\%) dans cette étude que dans les précédentes études d'enregistrement (14\%), peut-être du fait de la sélection de patients plus jeunes dans cette étude [3]. Au total, les facteurs de réponse optimale au traitement par ITK ne sont pas tous bien compris en dehors de l'apparition de mutations de résistance (tolérance, métabolisme, observance, immunologiques, etc.) et nécessitent d'être étudiés afin d'adapter au mieux le traitement à chaque patient. ]

\section{Références}

[1] Jain P, Kantarjian H, Nazha A, et al. Early responses predict better outcomes in patients with newly diagnosed chronic myeloid leukemia results with four tyrosine kinase inhibitor modalities. Blood 2013 ; 121 : 4867-74.

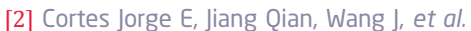
Dasatinib vs. imatinib in patients with chronic myeloid leukemia in chronic phase (CML-CP) who have not achieved an optimal response to 3 months of imatinib therapy : the DASCERN randomized study. Leukemia 2020 ; 34 : 2064-73.

[3] Kantarjian HM, Shah NP, Cortes JE, et al. Dasatinib or imatinib in newly diagnosed chronic phase chronic myeloid leukemia : 2-year follow-up from a randomized phase 3 trial (DASISION). Blood 2012 ; 119 : 1123-9.

\section{Prise en charge de la splénomégalie chez les patients atteints de myélofibrose : quelles options en 2020 ?}

\author{
Laly Nsiala \\ Jose Miguel Torregrosa Diaz \\ améliorer la qualité de vie de ces \\ patients.
}

a myélofibrose (MF) est un syndrome myéloprolifératif, caractérisée par une fibrose médullaire avec risque d'hématopoïèse extramédullaire au cours de l'évolution. Un des symptômes les plus fréquents est la splénomégalie (SMG), avec $38 \%$ des patients présentant une rate $>10 \mathrm{~cm}$ de débord costal. Elle est responsable de cytopénies et d'absence de rendement transfusionnel en lien avec une séquestration splénique, mais aussi de douleurs de l'hypocondre gauche, d'une perte de poids par satiété précoce en lien avec une compression gastrique et d'une hypertension portale. La diminution de la taille de la rate est donc un enjeu majeur pour

\section{Traitements validés dans la myélofibrose}

Les traitements classiquement essayés pour la SMG comprennent (figure 1) :

- l'hydroxyurée, efficace dans $\sim 40 \%$ de patients selon les séries, mais avec une toxicité hématologique limitante,

- l'interféron $\alpha$ (IFN $\alpha$ ), sans efficacité dans ce contexte,

- le melphalan à faibles doses, qui comporte surtout un risque élevé de néoplasies secondaires, pour une efficacité médiocre,

- les médicaments immunomodulateurs (thalidomide et lénalidomide), qui n'ont pas montré d'efficacité sur la SMG mais surtout sur l'anémie dans un sous-groupe de patients.

\section{Ruxolinitib}

Le ruxolitinib est un inhibiteur de la Janus kinase 2 (JAK2) indiqué dans le traitement des MF de risque intermédiaire et élevé suite aux résultats des études de phase III COMFORT [2]. Dans COMFORT I, qui comparait l'efficacité du ruxolinitib contre placebo, 41,9\% des patients traités par ruxolitinib ont obtenu une réduction du volume de la rate (RVR) $\geq 35 \%$ (versus $0,7 \%$ ). Des résultats similaires ont été obtenus dans COMFORT II (ruxolitinib versus meilleur traitement disponible), avec $53,4 \%$ des patients traités 